\title{
CHANGES IN THE STRUCTURAL CHARACTERISTICS OF PLATELETS AND LEUKOCYTES IN PATIENTS WITH ARTERIAL HYPERTENSION UNDER THE INFLUENCE OF A HYDROGEN SULFIDE DONOR
}

\author{
Alexander Kravchuk \\ Department of blood circulation physiology ${ }^{1}$ \\ k0677569851@gmail.com \\ Kateryna Rozova \\ Department of hypoxia ${ }^{1}$ \\ erozova@ukr.net \\ ${ }^{1}$ Bogomolets Institute of Physiology of the National Academy of Sciences of Ukraine \\ Akademika Bogomoltsya str., 4, Kyiv, Ukraine, 01601
}

\begin{abstract}
The aim of the research. The influence of hydrogen sulphide donor on some structural characteristics of platelets and leukocytes in patients with arterial hypertension was studied.

Materials and methods. The survey involved patients (men) with hypertension aged 30-60 years, who were divided into 2 age groups: $30-40$ years and 40-60 years. Control groups (healthy men without signs of hypertension) were randomized by age. Electron microscopic and morphometric research methods were used in the investigation.

Results. Electron microscopic and morphometric evaluation of the structure of these cells has shown that it undergoes significant changes depending on patient's age. In the persons of the older age group, the cells contained a reduced number of dense $\delta$-granules and increased - $\alpha$-granules, which may indicate an impaired serotonin balance and increased susceptibility to thrombogenesis. The younger age group presented structural changes in platelets in a reversed order. Adding hydrogen sulphide donor at a dose of $400 \mathrm{mg}$ per day (phytopreparation Full Spectrum Garlic - Swanson Health Products, USA) to the traditional therapy of arterial hypertension leads to positive changes in the ultrastructure of the studied cells aimed at restoring the balance of serotonin, reducing the tendency to thrombogenesis, increasing the energy capacity of the mitochondrial apparatus.

Conclusion. The obtained results indicate that detected effects significantly depend on the patient's age (younger people respond more intensively) and the duration of the use of hydrogen sulphide donor (longer use is accompanied by more pronounced positive changes).
\end{abstract}

Keywords: arterial hypertension, hydrogen sulphide, platelet ultrastructure, leukocyte ultrastructure, mitochondria.

DOI: $10.21303 / 2504-5679.2020 .001288$

\section{Introduction}

In the scientific literature of recent years, considerable attention has been paid to low molecular weight signalling molecules, which are generally referred to $\mathrm{NO}$ and $\mathrm{CO}_{2}$. Much later, it was found that $\mathrm{H}_{2} \mathrm{~S}$ also referred to them $[1,2]$. It is now believed that the main biological effects of $\mathrm{H}_{2} \mathrm{~S}$ include the regulation of vascular tone, contractile activity of the myocardium, participation in long-term synaptic potentiation, pro- and anti-inflammatory reactions, regulation of insulin secretion, etc. $[3,4]$. Hydrogen sulphide has also been shown to have antioxidant and anti-apoptotic properties [5].

The cardiovascular system is subject to $\mathrm{H}_{2} \mathrm{~S}$ action. Like $\mathrm{NO}$ and $\mathrm{CO}$, it relaxes the smooth muscles of the blood vessels, reduces blood pressure and heart rate [6, 7]. There is a direct correlation between the severity of the disease and the level of hydrogen sulphide in blood: the more pronounced hypertension - the greater the hydrogen sulphide deficiency [8].

At present, it is substantiated that one of the leading causes of cardiovascular pathology is mitochondrial dysfunction (MD) - moreover, disorders in regulation of blood pressure occur on the background of progressive energy deficiency [9, 10]. In recent years, cardioprotective effect of $\mathrm{H}_{2} \mathrm{~S}$ has been proven, in particular in the case of arterial hypertension (AH) and it was 
found that inhibition by hydrogen sulphide opening of the mitochondrial pore plays an important role in the mechanism of the protective effect $[11,12]$. It should be noted that the development of MD leads to damage of the organelles membranes, to decrease in ATP synthesis, which is accompanied by a reduction in contractile activity and functional heart reserves and, as a consequence, to the decrease in its pumping function. Based on available data on the cardioprotective effect of hydrogen sulphide partially associated with its effect on mitochondria (MC), namely, $\mathrm{Ca}^{2+}-$ induced mitochondrial pore opening [11], it can be assumed that $\mathrm{H}_{2} \mathrm{~S}$ may influence the structural component of MD, namely, the ultrastructure of MC. However, at present, the issue of the mitochondrial apparatus structural changes in patients with $\mathrm{AH}$ has not been sufficiently studied. Possible ultrastructural prerequisites for the development of changes in vascular wall contractility and/or the risk of thrombus formation at the background of hypertension also require further investigation.

For the study of MD, the morphofunctional state of other cellular organelles and, indirectly, the state of the vascular wall, both in healthy humans and patients with pathology, virtually the only objects of the research (in the absence of surgery) are blood cells, in particular, platelets (P) and leukocytes (L). They are involved in many body processes, directly and indirectly related to hypertension: in the regeneration of tissues, the development of inflammatory and immune responses, and the provision of primary homeostasis. As for P, they contain a significant amount of different granules, which include glycoproteins, proteins, growth factors, ADP, ATP, calcium ions, serotonin, histamine, etc.; P and, in particular, L contain MC, which allows to study the structure and functions of these organelles under the development of pathological conditions of different genesis, including AH $[13,14]$.

The aim of the study was to investigate the effect of hydrogen sulphide donor on some of the structural characteristics of platelets and leukocytes in patients with arterial hypertension.

\section{Materials and methods of research}

The studies were conducted based on the Department of blood circulation physiology and Department of hypoxia Bogomoletz Institute of Physiology of the National Academy of Sciences of Ukraine.

All patients with arterial hypertension were conducted in the winter period of 2018-2019.

\section{1. Organization of research}

Study of the ultrastructure of blood cells (leukocytes and platelets) were performed in patients (men) with arterial hypertension (AH) aged 30-60 years, which were divided into 2 age groups:

1) $30-40$ years $(n=10$; mean age $-33.9 \pm 1.1$ year);

2) 40-60 years ( $n=10$; mean age $-50.6 \pm 1.7$ years).

The average blood pressure in persons of the 1st group was $140 / 90 \mathrm{~mm} \mathrm{Hg}$ and in patients of the 2 nd group $-160 / 100 \mathrm{~mm} \mathrm{Hg}$. Control groups $\left(\mathrm{K}_{1}(\mathrm{n}=10)\right.$ and $\mathrm{K}_{2}(\mathrm{n}=10)$ - healthy men without signs of hypertension) were randomized by age.

The examination of each patient was conducted in 4 stages: before the study; after the course of treatment of the underlying disease; after a 2-week course of specific treatment; after a month of specific treatment. The latter consisted of the exogenous (per os) addition to the therapy of a hydrogen sulphide donor - $400 \mathrm{mg}$ per day - contained in a phytopreparation Full Spectrum Garlic (Swanson Health Products, USA). The course intaking the phytopreparation was 30 days, during which patients were examined twice - after 2 weeks (relatively a short course of treatment - SCT) and at the end of treatment (relatively a long course of treatment - LCT).

\section{2. Methods of preparation for electron microscopic and morphometric studies}

Platelet-enriched (P) with the inclusion of leukocytes (L) plasma was obtained by centrifugation of whole blood an ambient temperature for $15 \mathrm{~min}$ at $120 \mathrm{~g}$ on a laboratory centrifu- 
ge T-30 (Ukraine). The plasma was gently separated from the precipitated cells and centrifuged at 2000g for 20 min using Vortecs Combispin FVL-2400N mini-centrifuge (Latvia) [15].

Sampling for electron microscopic examination was carried out in accordance with conventional methods for blood cells with double fixation of glutaraldehyde and OsO4, dehydration in alcohols of increasing concentration and filling in Epon-Araldite (reagents from Fluka, Switzerland \& Sigma, USA) [16]. Ultrathin sections 40-60 nm thick were contrasted with solutions of uranyl acetate and lead citrate (Sigma reagents, USA) and examined in a TEM-124c electron microscope (Ukraine).

Morphometric calculations were performed using the Image Tool (USA) computer program in 130-150 fields for each group of subjects.

\section{3. Statistical analysis}

The statistical processing of the obtained data was performed using the Microsoft Exel and OriginPro software using the Student's t test, given the coherence of the sample of the surveyed. Due to the large array of digital material according to the Shapiro-Wilk criteria, the obtained data were within the normal distribution law, the data are presented as mean \pm mean error $(\mathrm{M} \pm \mathrm{m})$ [17]. The differences between the mean values were considered statistically significant at $\mathrm{p}<0.05$.

\section{Results}

As noted, two types of blood cells - leukocytes and platelets were investigated. Since they perform excellent functions and have different ultrastructures, it is possible to evaluate the greater number of cellular structural pathogenetic factors that are involved in (or a consequence of) hypertension. To some extent, they can (directly or indirectly) characterize the mechanisms that are responsible for the development and course of this pathological process.

We found significant differences in the structural organization of $\mathrm{L}$ and $\mathrm{T}$ in persons with hypertension in different age groups examined before the start of the standard treatment process.

With regard to $P$, the persons of mature age had virtually no dense $\delta$-granules, but the number of transparent $\alpha$-granules appeared to be increased (Table 1). In addition, the latter were often large (>100 nm) and contained a considerable amount of protein debris. Single mitochondria (MX) were structurally saved.

Table 1

Characteristics of platelet ultrastructure in individuals with arterial hypertension $(\mathrm{M} \pm \mathrm{m})$

\begin{tabular}{|c|c|c|}
\hline Groups of surveyed & $\delta$-granules, unit/cell & $\alpha$-granules, units/cell \\
\hline Control (mean age $30.4 \pm 2.8$ years) & $6.4 \pm 0.5$ & $9.1 \pm 0.7$ \\
\hline Control (mean age $54.2 \pm 1.9$ years) & $8.6 \pm 0.7$ & $5.3 \pm 0.9$ \\
\hline \multicolumn{3}{|c|}{ Patients with arterial hypertension - mean age $33.9 \pm 1.1$ years } \\
\hline Before treatment & $9.3 \pm 0.5^{*}$ & $6.2 \pm 0.6^{*}$ \\
\hline After 2 weeks of standard treatment & $7.5 \pm 0.6 \bullet$ & $8.3 \pm 0.5 \bullet$ \\
\hline After a 2-week course of specific treatment & $6.9 \pm 0.7 \bullet$ & $7.4 \pm 0.8 \bullet$ \\
\hline After a month of specific treatment & $5.9 \pm 0.4 \bullet \#$ & $9.6 \pm 0.5 \bullet \#$ \\
\hline \multicolumn{3}{|c|}{ Patients with arterial hypertension - mean age $50.6 \pm 1.7$ years } \\
\hline Before treatment & $3.3 \pm 0.2 *$ & $9.7 \pm 0.5^{*}$ \\
\hline After 2 weeks of standard treatment & $4.7 \pm 0.5^{*}$ & $8.8 \pm 0.6^{*}$ \\
\hline After a 2-week course of specific treatment & $5.0 \pm 0.3 * \bullet$ & $7.9 \pm 0.4 * \bullet$ \\
\hline After a month of specific treatment & $6.6 \pm 0.4^{*} \bullet \#$ & $6.9 \pm 0.6^{* \bullet \# ~}$ \\
\hline
\end{tabular}

Note: ${ }^{*}-p<0.05$ relative to control group; $\bullet-p<0.05$ relative to the group before treatment; $\#-p<0.05$ relative to standard treatment group 
One of the important functions of $\mathrm{P}$ is their participation in the metabolism of serotonin (S), it is established that the number of dense $\delta$-granules depends on its concentration [18]. In addition to S, the $\delta$-granules contain ADP and ATP and also accumulate $\mathrm{Ca}^{2+}$. P is virtually the only blood cell in which $\mathrm{S}$ reserves (up to $95 \% \mathrm{C}$, which is determined in plasma) are concentrated, so its amount accumulated in the $\delta$-granules indirectly indicates an exchange of $\mathrm{S}$, which is likely to be reduced in the examined patients. This may indicate dysfunction of the serotonergic system, as well as evidence of a regulatory effects disorder of S on the contractile capacity of the vascular wall, since $S$ is directly involved in the regulation of vascular tone, which contributes to its growth. Such changes may be compensatory, aimed at reducing vascular tone in hypertension $[15,19]$.

The number of large $\alpha$-granules containing proteins and glycoproteins involved in blood clotting, proteolytic enzymes, etc., was found to be significantly higher in $\mathrm{P}$ than in healthy humans (Table 1). It can be assumed that a significant number of granules that exhibit anti-heparin platelet factor 4, platelet growth factor, thrombospondin, which promote $\mathrm{P}$ aggregation, indicate an increased risk of thrombosis in elderly patients with AH [20].

The study of $\mathrm{P}$ patients of a younger age showed that hypertension was accompanied by the inverse changes in the ultrastructure of P. A significant decrease in the number of $\alpha$-granules and an increase in the number of $\delta$-granules were observed (Table 1). The identified feature requires further studies, it can only be suggested that it is associated with both age-related changes in blood cells and the duration of $\mathrm{AH}$. MC as in the previous group of the surveyed was mostly without changes. The lack of detection of changes in the ultrastructure of $\mathrm{MC}$ in persons of both age groups is probably due to their small number in $\mathrm{P}$ and the inability to perform a comprehensive morphometric evaluation of organelles. Such an opportunity appears when studying the ultrastructure of $\mathrm{L}$ under conditions of the $\mathrm{AH}$.

In recent decades, energy deficiency at the cellular level has been considered as the main cause of the primary increase in blood pressure. Degradation of energy forming function of mitochondria due to disruption in the structure of the mitochondrial apparatus (MCA) is considered to be a source of tissue energy metabolic disorder [21].

When analysing some of the structural features of L MCA in patients with AH, it was found that they had a significant amount of MC that was structurally altered (especially in the older age group), but their total number was not significantly changed in relation with the control values (Table 2).

It should be noted that in addition to swelling and vacuolation of organelles, which is a nonspecific response to most negative endo- or exogenous impacts on cells, there was a pronounced activation of autophagy of MC (Fig. 1).

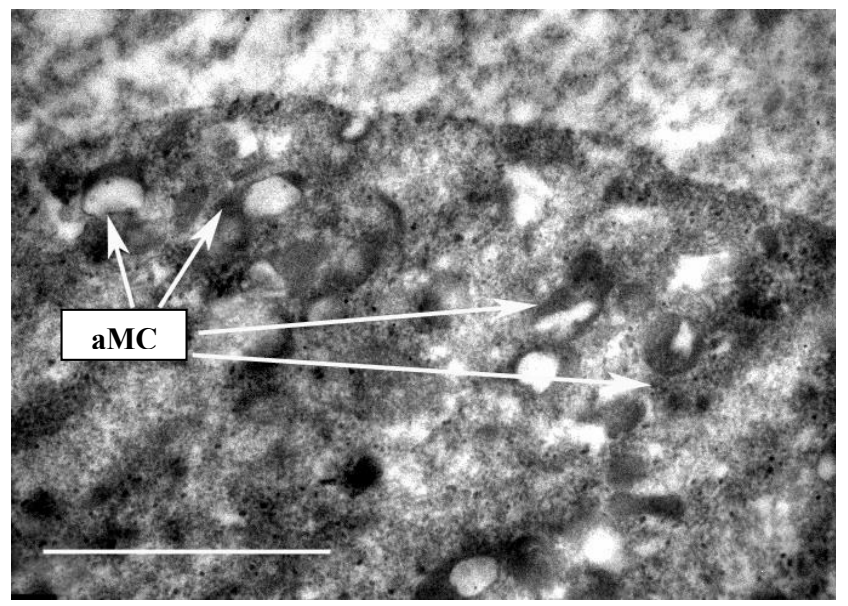

Fig. 1. Autophagy of mitochondria in the leukocyte of a patient with arterial hypertension: aMC - autophagy; Scale - $1 \mu \mathrm{m}$ 
Table 2

Some characteristics of mitochondrial apparatus ultrastructure of leukocytes in patients with arterial hypertension $(\mathrm{M} \pm \mathrm{m})$

\begin{tabular}{|c|c|c|c|}
\hline Groups of surveyed & $\begin{array}{c}\text { The number of mitochon- } \\
\text { dria, units } / 10 \mu \mathrm{m}^{2}\end{array}$ & $\begin{array}{l}\text { The number of structurally } \\
\text { damaged mitochondria, \% }\end{array}$ & $\begin{array}{l}\text { The average diameter of } \\
\text { mitochondria, } \mu \mathrm{m}\end{array}$ \\
\hline $\begin{array}{c}\text { Control } \\
\text { (mean age } 30.4 \pm 2.8 \text { years) }\end{array}$ & $11.3 \pm 0.5$ & $2.4 \pm 0.09$ & $0.54 \pm 0.07$ \\
\hline $\begin{array}{c}\text { Control } \\
\text { (mean age } 54.2 \pm 1.9 \text { years) }\end{array}$ & $9.5 \pm 0.6$ & $3.6 \pm 0.07$ & $0.61 \pm 0.04$ \\
\hline \multicolumn{4}{|c|}{ Patients with arterial hypertension - mean age $33.9 \pm 1.1$ years } \\
\hline Before treatment & $12.2 \pm 0.8$ & $26.2 \pm 2.4 * *$ & $0.69 \pm 0.05^{*}$ \\
\hline $\begin{array}{c}\text { After } 2 \text { weeks of standard } \\
\text { treatment }\end{array}$ & $12.7 \pm 0.9$ & $19.4 \pm 3.9^{* *} \bullet$ & $0.63 \pm 0.06^{*}$ \\
\hline $\begin{array}{l}\text { After a 2-week course of } \\
\text { specific treatment }\end{array}$ & $13.1 \pm 0.6^{*}$ & $18.6 \pm 2.6^{* *} \bullet \#$ & $0.58 \pm 0.03 \bullet$ \\
\hline $\begin{array}{l}\text { After a month of specific } \\
\text { treatment }\end{array}$ & $17.8 \pm 0.7 * \bullet$ & $12.8 \pm 4.2 * * \bullet \#$ & $0.47 \pm 0.04 \bullet \#$ \\
\hline \multicolumn{4}{|c|}{ Patients with arterial hypertension - mean age $50.6 \pm 1.7$ years } \\
\hline Before treatment & $10.3 \pm 0.7$ & $32.6 \pm 4.8^{* *}$ & $0.88 \pm 0.06^{*}$ \\
\hline $\begin{array}{c}\text { After } 2 \text { weeks of standard } \\
\text { treatment }\end{array}$ & $11.2 \pm 0.8$ & $28.8 \pm 5.3^{* *}$ & $0.79 \pm 0.05^{*}$ \\
\hline $\begin{array}{l}\text { After a 2-week course of } \\
\text { specific treatment }\end{array}$ & $11.9 \pm 0.6$ & $22.3 \pm 3.7 * * \bullet$ & $0.77 \pm 0.06^{*}$ \\
\hline $\begin{array}{c}\text { After a month of specific } \\
\text { treatment }\end{array}$ & $14.6 \pm 0.5 * \bullet$ & $20.2 \pm 1.6^{* * \bullet} \#$ & $0.70 \pm 0.03 \bullet \#$ \\
\hline
\end{tabular}

Note: ${ }^{*}-p<0.05$ relative to control group; $\bullet-p<0.05$ relative to the group before treatment; $\#-p<0.05$ relative to standard treatment group

\section{Discussion}

Because autophagy is thought to be a process that contributes not only to the death of damaged cells but also to their preservation (by preventing apoptosis by mitochondrial pathways) [22, 23], it can be assumed that in hypertension, regardless of the age of the patients, this mechanism is compensatory active, at least in blood cells.

When arterial hypertension MC swelling led to an increase in their average diameter (Table 2). However, if the patients of the 1st age group showed the increase of $27.8 \%$, the patients of the 2 nd group showed $44.3 \%$. This difference is important given that the growth in diameter of $\mathrm{MC}$ within $25-30 \%$ is considered to be an adaptive reaction aimed at enhancing the energy capacity of organelles caused by the activation of the ATP-dependent $\mathrm{K}+$ channel. More pronounced increased of $\mathrm{MC}$ diameter indicates to the possibility of their necrotic death and is often irreversible [24, 25].

Conducting traditional 2-week therapy was more effective for younger patients. Its most important results include: for $\mathrm{P}$ - the disappearance of differences from the control values of the number of both $\delta$ - and $\alpha$-granules; for $\mathrm{L}-$ a decrease of $35.1 \%$ in the number of structurally damaged MC. These changes relate to the 1st group of people with AH (Tables 1, 2). In patients of group 2, significant differences were found only in the increase in the number of $\delta$-granules (by $42.4 \%$ ), which may be important regarding the influence on the tone of the vascular wall. It can be assumed that the absence of a pronounced response of the studied indexes to traditional treatment is associated with both the duration of the disease and the small period of treatment (given the lack of pre-treatment of $\mathrm{AH}$ ). 
The peculiarity of the two-week specific treatment was expressed in that its most positive manifestations were observed relatively to MCA L (Table 2). In both groups of subjects with hypertension significantly reduced the number of structurally modified MC, not only in relation to the condition before treatment, but also in relation to a certain number of such MC after standard treatment (by $40.9 \%$ in the 1 st and by $29.1 \%$ - in 2 nd group). Such changes should be seen as an opportunity to increase the capacity of energy metabolism.

With respect to other morphometric parameters of $\mathrm{P}$ and $\mathrm{L}$, the addition to standard treatment donor of hydrogen sulphide increased (in group 1) or showed a tendency (in group 2) to approach the studied indicators to the levels inherent in healthy persons of the appropriate age (Tables 1, 2).

The most optimal for patients with $\mathrm{AH}$, in view of the obtained results, was a monthly course of specific treatment. The prolonged addition of phytopreparation Full Spectrum Garlic to traditional AH therapy improved all investigated $\mathrm{P}$ and $\mathrm{L}$ ultrastructure indices in both groups of patients (Tables 1, 2). Firstly, in the younger age group, the number of $\delta$ - and $\alpha$-granules in $\mathrm{P}$ did not differ significantly from the control one. Secondly, in the older age group, the number of $\delta$-granules increased twice as much relative to pre-treatment and by $40,4 \%$ relative to the determined after traditional therapy. That is, if we take into account the assumption based on available scientific data, that the number of $\delta$-granules in T correlates with the amount of serotonin $[14,26]$, and thus with the function of the serotonergic system, one can speak of a certain restoration of its balance, which is important, since the imbalance of the serotonin content in the blood causes significant fluctuations in vascular tone, which participates in hypertension development [27]. In this case, $\alpha$-granules were found to be $40.6 \%$ less than before treatment and $27.5 \%$ less than after traditional therapy. Such changes brought them closer to the control values, and may be evidence of a decrease in the predisposition to thrombosis in older patients with $\mathrm{AH}$. This effect is quite predictable since it has been shown that hydrogen sulphide is able to inhibit platelet aggregation [28].

Long-term treatment of $\mathrm{AH}$ with the use of a donor $\mathrm{H}_{2} \mathrm{~S}$ had a pronounced positive effect on the ultrastructure of MCA of leukocytes. Only with such a scheme of treatment there was a significant increase in the total amount of MC (Table 2): in persons of the younger age group - by $57.5 \%$, and in the older age group - by $53.7 \%$. At present there are few data on the influence of $\mathrm{H}_{2} \mathrm{~S}$ the total number of $\mathrm{MC}$ in cells. However, it is believed that $\mathrm{H}_{2} \mathrm{~S}$ exerts different effects on the number, function, and dynamics of MC depending on the used dose [29]. Probably the presumed concentration is effective for increasing the number of organelles.

A further decrease in the number of structurally damaged MC was also detected (Table 2). In 1st group of patients, the reduction was 2-fold relative to the condition before treatment and $51.6 \%$ relative to the traditional treatment; in patients of 2 nd group - in relation to the state before treatment - by $61.4 \%$, in comparison with traditional treatment - by $39.6 \%$. In younger patients the changes were more pronounced due to the greater adaptive capacity associated with age, the number of altered MC (including in the state of autophagy) was significantly reduced, which, together with the increase in their total quantity, had to significantly optimize energy metabolism.

There is another issue to be considered. The latest studies show that serotonin has an active effect on cell biology [30]. With regard to neurons, it activates the formation of new mitochondria and, at the same time, reduces the level of reactive oxygen species; it triggers enhanced ATP formation and prevents the development of oxidative stress. It can be assumed that a similar influence of greater or lesser degree of expression $\mathrm{H}_{2} \mathrm{~S}$ can exert in other cells. Therefore, restoring the balance of this mediator may indirectly affect on the formation of MC. In addition, it has been shown that even small concentrations of hydrogen sulphide can prevent mitochondrial damage. Thus, the ultrastructural changes in MCA of L may be due to both direct and indirect effects of $\mathrm{H}_{2} \mathrm{~S}$ due to the action of hydrogen sulphide on the serotonin balance.

Study limitations. Despite the positive results which we have obtained under the use of hydrogen sulphide donor in the treatment of hypertension, it should be noted that this effect is significantly dependent on the age of the patient and of the duration of treatment. In addition, it is shown that a significant increase its concentration in blood to millimolar values produces cytotoxic action, 
causes the activation of free radical processes, that is, instead of antioxidant action can have the opposite effect. In addition, endogenous hydrogen sulphide is involved in the pathogenesis of type 1 diabetes. Also it has been shown experimentally that $\mathrm{H}_{2} \mathrm{~S}$ is capable of producing both anti- and pro-inflammatory effects. Therefore, it should be emphasized that the use of exogenous hydrogen sulphide donors for therapeutic purposes should be strictly dosed.

Prospects for further researches. Thus, although the obtained results indicate a positive effect of $\mathrm{H}_{2} \mathrm{~S}$ on the ultrastructure and, consequently, the function of blood cells, in particular platelets and leukocytes, nevertheless require further research for the development of new effective ways of increasing $\mathrm{H}_{2} \mathrm{~S}$ content in patients with hypertension.

\section{Conclusions}

The results obtained enable us to draw the following conclusions.

1. The ultrastructure of platelets and leukocytes under arterial hypertension undergoes significant changes related to the patient's age.

2. The morphometric evaluation of platelets showed that the cells of the elderly patients contain a reduced number of dense $\delta$-granules and increased $-\alpha$-granules, which may indicate a disruption of serotonin balance and increased susceptibility to thrombogenesis. The younger age group presented reversed structural changes in platelets.

3. It is shown that Adding hydrogen sulphide donor at a dose of $400 \mathrm{mg}$ per day (phytopreparation Full Spectrum Garlic, Swanson Health Products, USA) to the traditional therapy of arterial hypertension leads to positive changes in the ultrastructure of the investigated cells aimed at restoring the balance of serotonin, reducing the tendency to thrombogenesis, increasing the energy capacity of the mitochondrial apparatus. These effects are significantly dependent on the patients' age (younger people with hypertension respond more intensively) and on the duration of intake of the hydrogen sulphide donor (a longer use is accompanied by more pronounced positive changes).

\section{Conflict of interest}

The authors declare there is no conflict of interest.

\section{References}

[1] Gadalla, M. M., Snyder, S. H. (2010). Hydrogen sulfide as a gasotransmitter. Journal of Neurochemistry, 113 (1), $14-26$. doi: http://doi.org/10.1111/j.1471-4159.2010.06580.x

[2] Berezovsky, V. Ya., Plotnikova, L. M. (2012). Hydrogen sulfide and its role in the regulation of vascular tone. Medical hydrology and rehabilitation, 10 (1), 4-10.

[3] Du, S., Jin, H., Bu, D., Zhao, X., Geng, B., Tang, C., Du, J. (2008). Endogenously generated sulfur dioxide and its vasorelaxant effect in rats1. Acta Pharmacologica Sinica, 29 (8), 923-930. doi: http://doi.org/10.1111/j.1745-7254.2008.00845.x

[4] Berezovsky, V. A., Plotnikova, L. M. (2013). The role of endogenous hydrogen sulfide in the regulation of physiological functions. Medical hydrology and rehabilitation, 11 (1), 117-122.

[5] Sukmansky, O. I. (2017). Sulfur-containing gaseous signaling molecules. Fiziolohichny̌̌ Zhurnal, 63 (6), 106-117. doi: http:// doi.org/10.15407/fz63.06.106

[6] Yang, G., Wang, R. (2015). H2S and Blood Vessels: An Overview. Handbook of Experimental Pharmacology, 85-110. doi: http://doi.org/10.1007/978-3-319-18144-8_4

[7] Yoo, D., Jupiter, R. C., Pankey, E. A., Reddy, V. G., Edward, J. A., Swan, K. W. et. al. (2015). Analysis of cardiovascular responses to the H2S donors Na2S and NaHS in the rat. American Journal of Physiology-Heart and Circulatory Physiology, 309 (4), H605-H614. doi: http://doi.org/10.1152/ajpheart.00171.2015

[8] Barna, O. M., Snigir, N. V. (2017). Hydrogen is an important signaling molecule in the pathogenesis of cardiovascular disease: potential therapeutic effects. Drugs of Ukraine, 4, 23-25.

[9] Postnov, Yu. V. (2000). The development of the membrane concept of pathogenesis of primary hypertension (impaired mitochondrial function and energy deficiency). Cardiology, 10, 4-12.

[10] Kolesnik, M. Yu., Belenichev, I. F., Dzyak, G. V., Checkman, I. S. (2012). Features of functioning of myocardial mitochondria in rats with spontaneous hypertension (SHR) on the background of experimental sugar diabetes and atherosis. Zaporizhzhya medical journal, 2, 26-30. 
[11] Strutyns'ka, N., Dorofeieva, N., Vavilova, H. et. al. (2012). Increase in the sensitivity of the mitochondrial permeability transition pore opening to $\mathrm{Ca}^{2+}$ in heart of spontaneous hypertensive rat. Fiziolohichnyĭ Zhurnal, 58 (6), 3-8. doi: http://doi.org/ $10.15407 /$ fz 58.06 .003

[12] Strutyns'ka, N., Dorofeieva, N., Vavilova, H., Sahach, V. (2013). Hydrogen sulfide inhibits Ca(2+)-induced mitochondrial permeability transition pore opening in spontaneously hypertensive rats. Fiziolohichnyĭ Zhurnal, 59 (1), 3-10. doi: http://doi.org/ $10.15407 /$ fz59.01.003

[13] Kolosova, E. N., Vasilenko, I. A., Kovaleva, L. G. (2011). Assessment of morphofunctional condition of platelets of patients with idiopathic thrombotcytopenic purpura by method of vital computer morphometry. Bulletin of the Siberian Branch of Russian Academy of Medical Sciences, 31 (2), 58-63.

[14] Kostyuk, K. S. (2020) Quantitative and qualitative change of leukocytes in pathological processes. Available at: https:// www.ncgb.by/index.php/gazeta-ncgb-meterial/41-gazeta-statiy/1055-kolichestvennoe-i-kachestvennoe-izmenenie-lejkotsitov-pri-patologicheskikh-protsessakh

[15] Filippova, O. I., Koloskov, A. V., Capital, A. A. (2012). Methods for the study of the functional activity of platelets (literature review). Transfusiology, 13 (2), 493-514.

[16] Karupu, V. Ya. (1984). Electron microscopy. Kiyv: Vyshcha shkola, 208.

[17] Lukyanova, E. M., Antipkin, Yu. G., Chernyshov, V. P., Vykhovanets, E. V. (2002). Methods of statistical processing of medical information in scientific research. Kiyv: Planeta lyudey, 200.

[18] Clauser, S., Cramer-Bordé, E. (2009). Role of Platelet Electron Microscopy in the Diagnosis of Platelet Disorders. Seminars in Thrombosis and Hemostasis, 35 (2), 213-223. doi: http://doi.org/10.1055/s-0029-1220329

[19] Matvinko, Yu. (2017). Nervous system and arterial hypertension - pathogenetic interactions. Medicine of world. Available at: http://msvitu.com/archive/2017/august/article-4.php?print=1

[20] Goncharuk, I. V., Artemova, N. E., Bugrova, O. V. (2010). Relationship of platelet changes and some immunological parameters in systemic lupus erythematosus. Conference materials of Orenburg State Medical University, 75-78.

[21] Runikhin, A. Yu., Poryadin, G. V., Savchuk, V. I. (2011). Molecular and cellular mechanisms of the pathogenesis of primary hypertension. Bulletin of the Russian State Medical University, 6, 5-10.

[22] Kovaleva, O. V., Shitova, M. S., Zborovskaya, I. B. (2014). Autophagy: cell death or a way to survive? Clinical oncohematology. Basic research and clinical practice, 7 (2), 103-113. doi: https://doi.org/10.17116/repro201723481-86

[23] Mijaljica, D., Prescott, M., Devenish, R. J. (2011). Microautophagy in mammalian cells: Revisiting a 40-year-old conundrum. Autophagy, 7 (7), 673-682. doi: http://doi.org/10.4161/auto.7.7.14733

[24] Solodovnikova, I. M., Saprunova, V. B., Bakeeva, L. E., Yaguzhinsky, L. S. (2006) Dynamics of changes in the mitochondrial ultrastructure of cardiomyocytes of an isolated rat myocardium during prolonged incubation under anoxia. Cytology, 48 (10), 848-855.

[25] Mironova, G. D., Rozova, E. V., Belosludtseva, N. V., Man'kovskaya, I. N. (2019). Dynamic Restructuring of the Myocardial Mitochondria in Response to Uridine Modulation of the Activity of Mitochondrial ATP-Dependent Potassium Channel under Conditions of Acute Hypoxic Hypoxia. Bulletin of Experimental Biology and Medicine, 166 (6), 806-810. doi: http://doi.org/ 10.1007/s10517-019-04445-9

[26] Gildea, J. J., Wang, X., Shah, N., Tran, H., Spinosa, M., Van Sciver, R. et. al. (2012). Dopamine and Angiotensin Type 2 Receptors Cooperatively Inhibit Sodium Transport in Human Renal Proximal Tubule Cells. Hypertension, 60 (2), $396-403$. doi: http://doi.org/10.1161/hypertensionaha.112.194175

[27] Sadykova, D. I., Nigmatullina, R. R., Aflyatumova, G. N. (2015). The role of serotonergic system in cardiovascular diseases development in children. Kazan Medical Journal, 96 (4), 665-669. doi: http://doi.org/10.17750/kmj2015-665

[28] Varaksin, A. A., Pushchina, E. V. (2012). The value of hydrogen sulfide in the regulation of organ functions. Pacific Medical Journal, 2, 27-36.

[29] Qiao, P., Zhao, F., Liu, M., Gao, D., Zhang, H., Yan, Y. (2017). Hydrogen sulfide inhibits mitochondrial fission in neuroblastoma N2a cells through the Drp1/ERK1/2 signaling pathway. Molecular Medicine Reports, 16 (1), 971-977. doi: http://doi.org/ 10.3892/mmr.2017.6627

[30] Fanibunda, S. E., Deb, S., Maniyadath, B., Tiwari, P., Ghai, U., Gupta, S. et. al. (2019). Serotonin regulates mitochondrial biogenesis and function in rodent cortical neurons via the 5-HT2A receptor and SIRT1-PGC-1 $\alpha$ axis. Proceedings of the National Academy of Sciences, 116 (22), 11028-11037. doi: http://doi.org/10.1073/pnas.1821332116 